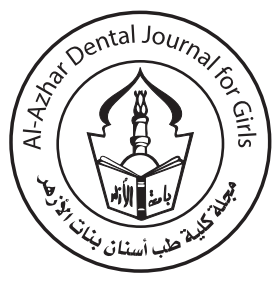

\title{
Effect of Diode Laser and Iontophoresis with Aqueous Extract of Nigella sativa and Fluoride-Based-Agent on Dentin Permeability
}

\section{Codex : 06/22.01 \\ azhardentj@azhar.edu.eg \\ http://adjg.journals.ekb.eg \\ DOI: $10.21608 /$ adjg.2021.57775.1329 \\ Restorative Dentistry \\ (Removable Prosthodontics, Fixed \\ Prosthodontics, Endodontics, Dental Biomaterials, Operative Dentistry)}

Aisha N. Zahran ${ }^{1 *}$, Maha A. Niazy ${ }^{2}$, Asmaa A. Mosleh ${ }^{3}$

\section{KEYWORDS}

Nigella sativa, Fluoride, Iontophoresis, Diode laser, Dentin permeability

\begin{abstract}
Purpose: Evaluation of the effect of diode laser and iontophoresis with aqueous extract of Nigella sativa and fluoride-based-agent on dentin permeability. Materials and Methods: Thirty-six lower premolars were used to prepare 72 samples and were randomly divided into three intervention groups 24 per group; Group A1 Samples were treated with aqueous extract of Nigella sativa, Group A2 Samples were treated with fluoride-based-agent, Group A3 Control group were left without treatment. The main groups were subdivided into two subgroups of 12 samples, according to surface treatments: Group B1 was treated with diode laser and Group B2 was treated with iontophoresis. Results: Permeability results revealed that there was statistically significant difference between the study groups with the lowest mean depth of dye penetration values was found in group samples treated with aqueous extract of Nigella sativa with iontophoresis. Conclusion: Aqueous extract of Nigella sativa, a fluoridebased-agent with the application of diode laser and iontophoresis on each was effective in reducing dentin permeability, being an aqueous extract of Nigella sativa the most effective when using with iontophoresis.
\end{abstract}

\section{INTRODUCTION}

Dentin permeability is an unfavorable condition in the dental field. Permeability of cervical dentin causes dentin hypersensitivity which was considered an oral health problem, affecting $4-57 \%$ of the population. Brannstrom's hydrodynamic theory proved that the pain sensation is caused by pulp nerves extremities stimulation through the occurrence

* A paper extracted from Masters thesis titled "Effect of Diode Laser and Iontophoresis with Aqueous Extract of Nigella Sativa and Fluoride-based Agent on Dentin Permeability"

1. Dentist at Specialized Medical Centers, Ministry of Health, Egypt.

2. Professor of Operative Dentistry Department, Faculty of Dental Medicine for Girls, Al-Azhar University, Cairo, Egypt.

3. Lecturer of Operative Dentistry Department, Faculty of Dental Medicine for Girls, Al-Azhar University, Cairo, Egypt

*Corresponding author email: dr.aisha.zahran@gmail.com 
of cervical enamel lesions, due to the indirect effect of dentinal fluid movement inside the tubules, after the exposure of dentinal tubules to the oral environmental ${ }^{(1)}$. Dentin permeability is generally measured during evaluations of the sealing ability to desensitize materials ${ }^{(2)}$.

The effect of laser on dentin permeability was assessed in several studies. Dentin minerals such as carbonate and phosphate absorb part of the energy when exposed to electromagnetic spectrum causing thermochemical ablation and provoking melting of the dentin tissue which leads to disarranging of dentin crystals ${ }^{(3)}$. The laser has a wavelength which when absorbed by the dentin cause a sufficient increase in temperature to get a melting effect hence, block the dentinal tubules. It has also been assumed that lasers coagulate the proteins inside the dentin structure and reduce the dentinal fluid movement ${ }^{(4)}$.

The iontophoretic process is considered another suggestion to reduce dentin permeability. The iontophoretic application into tooth structure results in the electrolysis of the calcium and phosphorous agents that in turn produce calcium and phosphorous ions that replace the hydroxyl group in tooth structure. The investigators claimed that in this way calcium and phosphorous ions are penetrating deeper layers of the tooth structure so its efficiency is the high and long-lasting duration ${ }^{(5)}$.

To minimize fluid movement, one strategy that has been used to overcome dentin permeability is patent dentinal tubules occlusion ${ }^{(6)}$. Using of therapeutic agents, such as fluoride-based-agentts and aqueous extract of Nigella sativa with diode laser and iontophoresis can promote the occlusion of the dentinal tubules. Fluoride-based-agents decrease the permeability of dentin by calcium fluoride crystals precipitating inside the dentinal tubule. Although fluoride's contribution in treating dentin hypersensitivity is well studied, there are still some high caries cases in which fluoride inadequately manages, so there is still a need for new strategies ${ }^{(7,8)}$.
Thousands of years ago, natural products have been utilized in folk medicine for so many purposes. Among them, the aqueous extract of the Nigella sativa plant has attracted interest due to its harmless nature and innumerable biological activities. Aqueous extract of Nigella sativa (Black seed) (Habatul Baraka) plant is used for eradicating various dangerous diseases. In this study, an aqueous extract of Nigella sativa is used for reducing dentinal permeability as Nigella sativa contains heavy minerals and metals, hence it can achieve dentinal tubules occlusion. Aqueous extract of Nigella sativa contains numerous inorganic elements as calcium, phosphorous, sodium, potassium, and iron ${ }^{(5)}$. It was found that aqueous extract of Nigella sativa's mineral content was $4.20 \%$ and that calcium and phosphorous ions concentration values in $100 \mathrm{gm}$ were $570 \mathrm{mg}$ and $543 \mathrm{mg}$ respectively which were followed by potassium ${ }^{(9)}$.

Whatever the effect of diode laser on dentin permeability is better than iontophoresis when used with aqueous extract of Nigella sativa and fluoridebased-agentt or not. Thus, the null hypothesis of the study is that: there is no association between diode laser and iontophoresis with aqueous extract of Nigella sativa and fluoride-based-agent in dentin permeability reduction. Therefore, the current study was designed to compare the effect of diode laser and iontophoresis using fluoride-based-agent and aqueous extract of Nigella sativa in treating dentin permeability.

\section{MATERIAL AND METHODS}

\section{Preparation of Aqueous extract of Nigella sativa:}

One hundred grams of seeds were ground, boiled in $1000 \mathrm{ml}$ water for $15 \mathrm{~min}$, and filtered through Whatman ${ }^{\circledR}$ qualitative filter paper No.1. Freeze drying of the filtrate was done by using a lyophilizer where water was removed after the freezing process and placed under a vacuum, allowing the ice to change directly from solid to vapor without passing through a liquid phase ${ }^{(10)}$. The aqueous extract was frozen for 24 hours at $-20^{\circ} \mathrm{C}$. 


\section{Sample grouping}

Thirty-one human lower premolars were used in this study to prepare sixty-two samples for the dentin permeability "dye penetration" test and additional ten teeth were used to prepare ten samples that were used for Scanning Electron Microscopic examination (SEM). A total of seventy-two samples were used in the study procedures which were randomly divided into three main groups of twentyfour per each, according to treatment agent applied: Group A1 Samples were treated with aqueous extract of Nigella sativa, Group A2 Samples were treated with a fluoride-based-agentt, Group A3 Control group were left without treatment. Group A1 and Group A2 were subdivided into two subgroups of twelve samples, according to surface treatments that used: B1 treated with diode laser and B2 treated with iontophoresis. In each subgroup, ten samples were used for dentin permeability (dye penetration test) and two samples were used for SEM examination.

\section{Preparation of specimens}

\section{Preparation of specimens for dentin permeability (dye penetration) test:}

Sixty-two teeth samples were used for the dye penetration test. Each tooth was sectioned longitudinally, in mesiodistal direction, into two parts (buccal and lingual), Cavities $2 \mathrm{~mm}$ wide and $0.8 \mathrm{~mm}$ deep were then prepared on the cervical region $1 \mathrm{~mm}$ coronal to the gingival line using two round burs \# 2 and \#6 carbide bur on the low-speed handpiece, with copious coolant ${ }^{(11)}$. Calliper was used to measure the diameters of the used burs. The cervical cavity was first prepared with the smaller bur (\#2) followed by the larger one to the same depth previously made by the first bur. Before the treatments, the samples were treated with $37 \%$ phosphoric acid gel for three minutes (after the acid application, rinsing, and drying for 30 seconds each) ${ }^{(12)}$ for the smear layer removal and the dentinal tubules opening and representing maximum dentin permeability.

\section{Preparation of specimens for scanning electron microscope (SEM) examination:}

Ten teeth were used for examination with SEM, firstly, the superficial dentin and occlusal enamel got removed by the double-faced diamond disc in the cutting machine, causing a flat mid-coronal dentin surface exposure, and creating a crown segment with $5 \mathrm{~mm}$-thickness ${ }^{(13)}$. Secondly, the pulp tissues were carefully removed (without dentin walls touching) and using deionized water to thoroughly rinse the pulp chamber. To polish the occlusal side of the crown segments (flat dentin side) the bulk of the smear layer was removed, and then each tooth thin remnant smear layer was etched with $37 \%$ phosphoric acid gel for three minutes ( after application of the acid, rinsing and drying for 30 seconds each) to keep the dentinal tubules patent ${ }^{(12)}$.

\section{Application of treatment agents:}

\section{Application of aqueous extract of Nigella sativa and fluoride-based-agentts:}

\section{- Iontophoresis treatment group:}

The freshly prepared aqueous extract of Nigella sativa and fluoride-based-agentt were applied to the prepared cervical cavities and dentin surfaces separately using fine size micro brush. Specimens were subjected to iontophoresis at $1.5 \mathrm{~mA}$ output current for three minutes and then removed using cotton pellets ${ }^{(14)}$.

\section{- Laser treatment group:}

The freshly prepared aqueous extract of Nigella sativa and fluoride-based-agentt were applied to the prepared cervical cavities and dentin surfaces separately using fine size micro brush. Using $980 \mathrm{~nm}$ diode laser at $0.5 \mathrm{w} / \mathrm{PW}(62.2 \mathrm{~J} / \mathrm{cm} 2)$ for 30 seconds on non-contact mode, specimens were lased ${ }^{(14)}$.

\section{Assessment of Dentin permeability:}

5\% Evans blue dye solution was applied to the cavities using plastic syringe and left for $20 \mathrm{~min}$, 
then rinsed and air dried. Cervical dentin samples were sectioned longitudinally (bucco-lingual direction) through the center of the cavities using double faced diamond disc in cutting machine. The samples were photographed by stereomicroscope at40 $\times$ magnification and images calculated by micrometer. The image analysis software by image $\mathrm{J}$ was used to calculate the depth of penetration from cavity toward pulp chamber at three points ${ }^{(15)}$.

\section{RESULTS}

Permeability results showed that there was a high statistically significant difference between the study groups in both dye penetration test (table 1) and scanning electron microscope examination (Fig. 1A-E). Comparing the mean values of the degree of occlusion for the tested groups in micrometer, results revealed that the lowest mean values (lowest dye penetration depth) $(256.00 \pm 20.66)$ $(\mathrm{p}<0.001)$ were recorded for aqueous extract of Nigella sativa with iontophoresis group followed by fluoride with iontophoresis group $(768.33 \pm 14.72)$ ( $p<0.001)$, followed by fluoride with diode laser $(1744.44 \pm 41.26)(\mathrm{p}<0.001)$ and aqueous extract of Nigella sativa with diode laser (3362.22 \pm 93.11$)$ $(\mathrm{p}<0.001)$, while the highest mean value (highest dye penetration depth) was recorded for the control group $(6581.00 \pm 39.00)(\mathrm{p}<0.001)$.

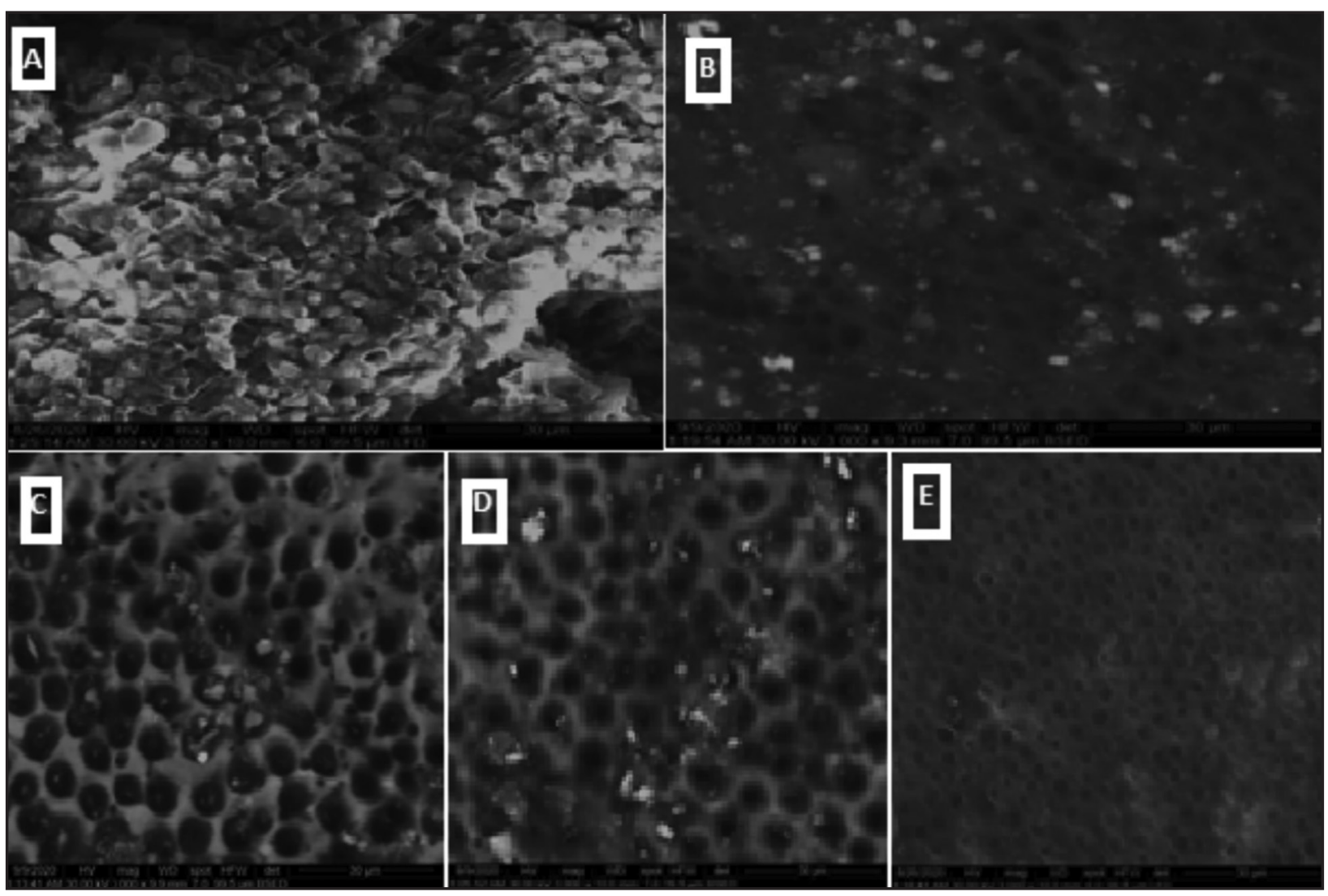

Figure (1) SEM photomicrographs of different groups A) Aqueous extract of Nigella sativa with iontophoresis, B) Fluoride-basedagentt with iontophoresis, C) Fluoride-based-agentt with diode laser, D) Aqueous extract of Nigella sativa with diode laser, E) control group (treated with phosphoric acid etchant gel) 
Table (1): Mean \pm standard deviation (SD) of dye penetration depth $(\mu \mathrm{m})$ for different groups

\begin{tabular}{cccccc}
\hline Material & Surface treatment & Mean & Std. Deviation & Rank & p-value \\
\hline \multirow{2}{*}{ Nigella sativa (A1) } & Diode laser (B1) & 3362.22 & 93.11 & $30.00^{\mathrm{AB}}$ & \\
\cline { 2 - 5 } & Iontophoresis (B2) & 256.00 & 20.66 & $5.50^{\mathrm{D}}$ & \\
\hline \multirow{2}{*}{ Fluoride (A2) } & Diode laser (B1) & 1744.44 & 41.26 & $21.00^{\mathrm{BC}}$ & \multirow{2}{*}{$\mathbf{0 . 0 0 1 *}$} \\
\cline { 2 - 5 } & Iontophoresis (B2) & 768.33 & 14.72 & $39.50^{\mathrm{CD}}$ \\
\hline
\end{tabular}

Different superscript letters indicate a statistically significant difference within the same vertical column*; significant $(p \leq 0.05)$ ns; non-significant $(p>0.05)$

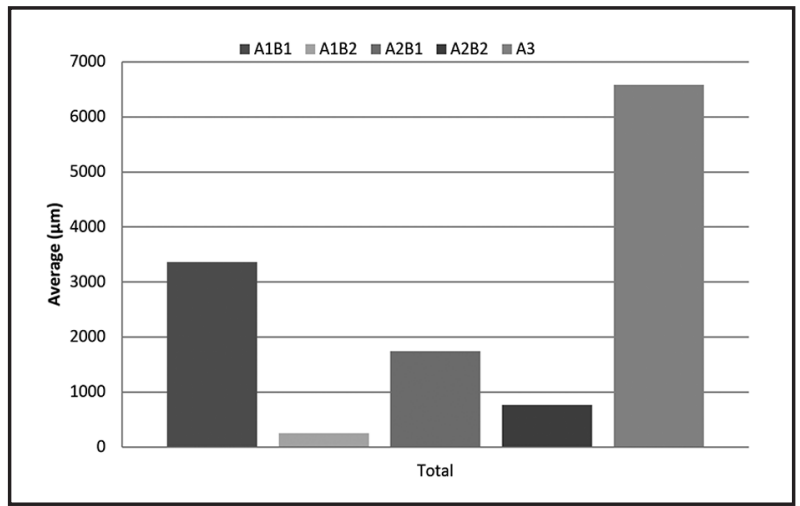

Figure (1) Bar chart showing average dentin permeability $(\mu \mathrm{m})$ for different group

\section{DISCUSSION}

Although, dentin permeability is essential to enable nutrients exchange to and from the pulp through the odontoblastic process ${ }^{(13)}$; this permeability may cause dentin sensitivity. Also, dentinal tubules have an essential role in stimuli and irritants delivery to the pulp. The transducing mechanism of certain stimuli of dentin is hypothesized by the hydrodynamic theory to be caused by the fluid flow through the tubules, also it was found that dentin with a larger number of open and wide dentinal tubules on the surface are hypersensitive than closed and narrow one ${ }^{(16)}$.

In recent years, many research groups have attempted to decrease dentin permeability by chemical agents such as sodium fluoride, arginine, and calcium carbonate ${ }^{(17)}$. There is an urgent need for a use of herbal materials and the efficacy and safety of herbal remedies. These agents effectively decrease the dentin permeability to some extent superficially with short-lived, limited depth infiltration, and their effect diminished during teeth brushing, food chewing, dissolution by saliva, or consumption of acidic beverage ${ }^{(18)}$. So using some surface treatments such as diode laser and iontophoresis in addition to desensitizing agents could be more effective for dentinal tubules sealing.

Aqueous extract of Nigella sativa was selected for this study, which acts as utilizing safe and effective natural materials that can block exposed dentinal tubules, reduce or inhibit the flow of tubular fluid, thus is an effective means for controlling dentin permeability ${ }^{(19)}$. Numerous inorganic elements such calcium, phosphorous, potassium, sodium, and iron range from (1.79\%-3.74 \%) was found in Nigella sativa ${ }^{(20)}$.

A fluorides-based agent was selected for this study since this fluoride compound in particular was found in several previous studies to provide an optimal dentinal tubule occlusion with reduction of dentin permeability ${ }^{(21)}$. Fluorides decrease the permeability, possibly by precipitation of insoluble calcium fluoride within the tubules ${ }^{(22)}$. In this study, fluoride gel was used to determine the desensitizing effect comparable to Nigella sativa aqueous extract, as fluoride is the most commonly used effective remineralizing agent, making it the reference material when new materials are evaluated ${ }^{(23,24)}$. The use of fluoride agents has been promoted 
because it increases the fluoride's time of action in contact with exposed dentin, thus reduction of dentin permeability ${ }^{(25)}$. The advent of lasers and iontophoresis gave us another option on dentin permeability.

The diode laser can provoke a melting effect with the dentine inorganic component re-crystallization resulting in dentinal tubules occlusion. Also, it has been found that laser treatment reduces sensitivity by the coagulation of protein of dentin organic components that lead to dentinal tubules sealing causing alterations on the exposed dentin surface ${ }^{(26)}$. In addition, the development of secondary dentin, which allows physiological occlusion of the dentinal tubules, is a bio stimulating influence ${ }^{(27)}$.

On the other hand, iontophoresis was selected for this study, as using some desensitizing materials with iontophoresis gave the best results for dentinal tubules occlusion comparable to using desensitizing materials alone. Iontophoresis was used to reduce dentin permeability in the early 1960s, thereby treating dentin hypersensitivity. Iontophoresis is a non-invasive procedure in which medications are delivered directly at the treatment sites with minimum adverse effects ${ }^{(28)}$. In the scientific literature, evidence on the use of iontophoresis with dental substances is still scarce. Some studies highlighted the positive effects of the use of certain desensitizing materials with iontophoresis provided dentinal tubule occlusion by allowing this ion to be transferred deeply to dentinal tubules ${ }^{(29)}$.

Analysis of the dentin permeability using dye diffusion in extracted premolars was deemed the appropriate objective of this study. At comparing the mean value of the degree of occlusion for the tested group, the results revealed that the lowest mean values (lowest dye penetration depth) were recorded for aqueous extract of Nigella sativa with iontophoresis group, it may be because the aqueous extract of Nigella sativa has several chemical compositions such as; volatile oil, fixed oil, protein, carbohydrates, alkaloids, saponins, amino and fatty acids, trace elements, heavy metals and minerals ${ }^{(30,31)}$. Moreover, Iontophoresis enhanced the penetration of those minerals deeply inside of dentinal tubules which leads to an increased lifetime of remineralization process and hence blocking of dentin permeability ${ }^{(29)}$. The highest mean value (highest dye penetration depth) was recorded for the control group where all the dentinal tubules were completely opened. This result of the degree of occlusion for the SEM observations matched the mean depth of dye penetration test results.

\section{CONCLUSION}

Under the conditions of the present study, it is concluded that all treatment materials used were effective in reducing the dentin permeability; with aqueous extract of Nigella sativa followed by iontophoresis being the most effective. Further treatment agents with iontophoresis could augment the effectiveness of desensitizing agents, and all the treatment materials produced micro-morphological changes of the dentin surface in terms of occluding the orifices of dentinal tubules to variable degrees.

\section{ACKNOWLEDGEMENT}

I would like to express my deepest gratitude and sincere appreciation to Dr. Mustafa Sharaf El-Deen, Assistant lecturer of clinical pharmacy, Mansoura university; who freely gave his time, efforts and experience along with continuous guidance during this work. Additional thanks to Dr. Menna-Allah Salem, lecturer of operative dentistry, Al-Azhar university; for her great help and precious advices.

\section{RECOMMENDATION}

Further in vivo studies should be done to evaluate the effect of iontophoresis on dentin hypersensitivity.

\section{CONFLICT OF INTEREST}

The authors declare that there is no conflict of interest.

\section{FUNDING}

No funding was received for this study. 


\section{REFERENCES}

1. Liu X, Tenenbaum H, Wilder R, Quock R, Hewlett E, Ren $\mathrm{Y}$ et al. Pathogenesis, diagnosis and management of dentin hypersensitivity: an evidence-based overview for dental practitioners. BMC Oral Health. 2020; 20:220.

2. Mathew M, Soni A, Khan M, Kauser A, Charan V, Akula $S$ et al. Efficacy of remineralizing agents to occlude dentinal tubules in primary teeth subjected to dentin hypersensitivity: In vitro SEM study. J Family Med Prim Care. 2020; 9:354-8

3. Sanches J, Faraoni J, Palma-Dibb R. Effect of desensitizing medications with and without diode laser treatment on dentin permeability and surface morphology. J Int Acad Periodontol. 2017; 19:57-64.

4. Rezazadeh F, Dehghanian P, Jafarpour D. Laser effects on the prevention and treatment of dentinal hypersensitivity: A systematic review. J Lasers Med Sci.. 2019; 10:1-11.

5. Hoobi N, Rzoqi M. Dissolution of inorganic phosphorous ion from teeth treated with different concentrations of aqueous extract of Nigella sativa (black seed) in comparison with sodium fluoride: An in vitro study. Int. J. Sci. Res. 2017; 6:78-96.

6. Yoshizaki K, Francisconi-Dos-Rios L, Sobral M, Aranha A, Mendes F, Scaramucci $T$ et al. Clinical features and factors associated with non-carious cervical lesions and dentin hypersensitivity. J. Oral Rehabil. 2017; 44:112-8.

7. Lucchese A, Bertacci A, Zanna S, Chersoni S, Manuelli $\mathrm{M}$, Visone A et al. In vitro effects of fluoride-based and desensitizing toothpastes on dentine permeability. J Biol Regul Homeost Agents. 2018; 32:51-8.

8. Ameen Sh, Niazy M, El-yassaky M, Jamil W. Clinical evaluation of nano-hydroxyapatite as dentin desensitizer. ADJ- for girls. 2018; 5:79-87.

9. Sultan M, Butt M, Anjum F, Jamil A, Akhtar S, Nasir M et al. Nutritional profile of indigenous cultivar of black cumin seeds and antioxidant potential of its fixed and essential oil. Pak. J. Bot. 2009; 41:1321-30.

10. Ali S, Meitei K. Nigella sativa seed extract and its bioactive compound thymoquinone: The new melanogens causing hyperpigmentation in the wall lizard melanophores. J. Pharm. Pharmaco. 2011; 63:741-6.

11. Braga T, Braga D, Moreno-Carvalho E, Bauer J, Turssi C. Calcium pre-rinse: Effect on permeability of dentin tubules by fluoride rinse. J Clin Exp Dent. 2019; 11:303-9.
12. Mosleh A, Niazy M, El-yassaky M. Clinical and laboratory evaluation of the efficacy of three different treatment modalities in management of dentin hypersensitivity. ADJfor girls. 2018; 5:129-34.

13. Zhou J, Chiba A, Scheffel D, Hebling J, Agee K, Niu L et al. Effects of a dicalcium and tetracalcium phosphatebased desensitizer: In vitro dentin permeability. PloS one. 2016; 11:e0158400.

14. Patil A, Varma S, Suragimath G, Abbayya K, Zope S, Kale V et al. Comparative evaluation of efficacy of iontophoresis with $0.33 \%$ sodium fluoride gel and diode laser alone on occlusion of dentinal tubules. J Clin Diagn Res. 2017; 11:123-6.

15. Pinto S, Pochapski M, Wambier D, Pilatti L, Santos F. In vitro and in vivo analyses of the effects of desensitizing agents on dentin permeability and dentinal tubule occlusion. J Oral Sci. 2010; 52:23-32.

16. Davari A, Ataei E, Assarzadeh H. Dentin hypersensitivity: Etiology, diagnosis and treatment; A literature review. J. Dent. (Shiraz). 2013; 14:136-45.

17. Bekes K, Heinzelmann K, Lettner S, Schaller H. Efficacy of desensitizing products containing $8 \%$ arginine and calcium carbonate for hypersensitivity relief in $\mathrm{MIH}-$ affected molars: an 8-week clinical study. Clin. Oral investig. 2017; 21:2311-17.

18. Martins V, Dacosta R, Pimenta L, Correia R, Cavalcanti A. Effect of surface protection on the permeability of eroded dentin. J. Conserv. Dent. 2018; 21:16-20.

19. Yuan P, Shen X, Liu J, Hou Y, Zhu M, Huang J et al. Effects of dentifrice containing hydroxyapatite on dentinal tubule occlusion and aqueous hexavalent chromium cations sorption: A preliminary study. PloS one. 2012; 7:e45283.

20. Atta M. Some characteristics of Niegella (Niegella Sativa L.) seed cultivated in Egypt and its lipid profile. Food Chem. 2003; 83:63-8.

21. Choi A, Jang I, Son S, Jung K, Park J. Effect of erosive and abrasive stress on sealing ability of different desensitizers: In-vitro study. PloS one. 2019; 14:e0220823.

22. Denucci G, Mantilla T, Amaral F, Basting R, Franca F, Turssi $C$ et al. Saliva with reduced calcium and phosphorous concentrations: Effect on erosion dental lesions. Oral Dis. 2018; 24:957-63.

23. Petersson L. The role of fluoride in the preventive management of dentin hypersensitivity and root caries. Clin. Oral Investig. 2013; 17:63-71. 
24. Ghafournia M, Tehrani M, Nekouei A, Faghihian R, Mohammadpour M, Feiz A, et al. In vitro evaluation of dentin tubule occlusion by three bioactive materials: A scanning electron microscopic study. Dent Res J. 2019; 16:166-71.

25. Lussi A, Buzalaf M, Duangthip D, Anttonen V, Ganss C, Joao-Souza $S$ et al. The use of fluoride for the prevention of dental erosion and erosive tooth wear in children and adolescents. Eur. J Paediatr. Dent. 2019; 20:517-27.

26. Al-khafaji Z, Awazli L, Al-Maliky M. Effects of diode laser $940 \mathrm{~nm}$ with and without $5 \%$ sodium fluoride white varnish with tri-calcium phosphate on dentin permeability: In vitro study. Iraqi J Laser. 2018; 17:17-25.

27. Marto C, Baptista P, Nunes T, Pimenta M,Abrantes A, Pires A et al. Evaluation of the efficacy of dentin hypersensitivity treatments: A systematic review and follow-up analysis. J. Oral Rehabil. 2019; 46:952-90.
28. Wei R, Simon L, Hu L, Michniak-Kohn B. Effects of iontophoresis and chemical enhancers on the transport of lidocaine and nicotine across the oral mucosa. Pharm. Res. 2012; 29:961-71.

29. Pauli M, Tabchoury C, Silva S, Ambrosano G, Lopez R, Leonardi $\mathrm{G}$ et al. Effect of iontophoresis on fluoride uptake in enamel with artificial caries lesion. Braz Oral Res. 2019; $33: 18-31$

30. El-Tahir K, Bakeet D. The Black seed Nigella sativa: Aplea for urgent clinical evaluation of its volatile oil. JTUMed Sc. 2006; 1:1-19.

31. Ahmad A, Husain A, Mujeeb M, Siddiqui NA, Damanhouri ZA, Bhandari A et al. Physicochemical and phytochemical standardization with HPTLC fingerprinting of Nigella sativa L. seeds. Pak J Pharm Sci. 2014; 27:1175-82. 